\title{
Synthesis of Ultrathin Perovskite Nanowires via a Postsynthetic Transformation Reaction of Zero- Dimensional Perovskite Nanocrystals
}

Hanjun Yang, Tong Cai, Lacie Dube, Katie Hills-Kimball, and Ou Chen*

Department of Chemistry, Brown University, Providence, RI 02912, USA

*Email: ouchen@brown.edu 


\section{Chemicals}

Cesium carbonate $\left(\mathrm{Cs}_{2} \mathrm{CO}_{3}, 99.9 \%\right)$, lead bromide $\left(\mathrm{PbBr}_{2}, 99.999 \%\right.$ trace metal basis), oleic acid (OA, technical grade, 90\%), oleylamine (OAm, technical grade, 70\%), 1-octadecene (ODE, technical grade, 90\%), hexanoic acid (HA, >99.5\%), octylamine (OctAm, 99\%) and trioctylamine (TOAm, 98\%) were obtained from Aldrich. Lead iodide ( $\left.\mathrm{PbI}_{2}, 98.5 \%\right)$ were obtained from Alfa Aesar. Hexane, toluene and ethyl acetate were obtained from Fisher Scientific. All chemicals were used as received without further purification.

\section{Experimental procedures}

\section{Synthesis of zero-dimensional (0D) $\mathrm{Cs}_{4} \mathrm{PbX}_{6}$ nanocrystals (NCs).}

The $0 \mathrm{D} \mathrm{Cs}_{4} \mathrm{PbBr}_{6}$ perovskite NCs were synthesized following a reported hot-injection method. ${ }^{1}$ Specifically, $\mathrm{PbBr}_{2}(73.4 \mathrm{mg}, 0.2 \mathrm{mmol})$ was added to a mixture solution of ODE $(10 \mathrm{~mL})$, oleic acid $(0.4 \mathrm{~mL})$ and oleylamine $(3 \mathrm{~mL})$, and then degassed at $80^{\circ} \mathrm{C}$ under vacuum for 1 hour. Then, $1.5 \mathrm{~mL}$ of cesium oleate solution $\left(0.4 \mathrm{~g} \mathrm{Cs} 2 \mathrm{CO}_{3}\right.$ reacted with $8 \mathrm{~mL}$ OA at $100^{\circ} \mathrm{C}$ for 30 min) were swiftly injected into the $\mathrm{PbBr}_{2}$ solution under nitrogen. The reaction was immediately quenched by submerging the flask into an ice-water bath. The resulting NCs were separated from the reaction solution by centrifuging at $8000 \mathrm{rpm}$ for 5 minutes. The NCs were redispersed in $10 \mathrm{~mL}$ hexane and purified twice using ethyl acetate. Finally, the purified $\mathrm{Cs}_{4} \mathrm{PbBr}_{6} \mathrm{NCs}$ were dispersed in $10 \mathrm{~mL}$ hexane for further use. A similar procedure was applied to the synthesis of $0 \mathrm{D} \mathrm{Cs} 4 \mathrm{PbI}_{6}$ perovskite $\mathrm{NCs}$ but at a lower reaction temperature $\left(60^{\circ} \mathrm{C}\right)$. The final product underwent only one round of ethyl acetate purification.

\section{Post-synthetic transformation reaction}

For a typical transformation reaction to obtain $\mathrm{CsPbBr}_{3}$ nanowires (NWs), $\mathrm{PbBr}_{2}(10 \mathrm{mg}, 0.027$ mmol) was added to a mixture solution of hexane $(5 \mathrm{~mL}), \mathrm{HA}(5 \mu \mathrm{L}, 0.04 \mathrm{mmol}), \mathrm{OA}(10 \mu \mathrm{L}$, $0.03 \mathrm{mmol})$ and OctAm $(10 \mu \mathrm{L}, 0.06 \mathrm{mmol})$ in a scintillation vial and heated to $50^{\circ} \mathrm{C}$. Then, $0.5 \mathrm{~mL} \mathrm{Cs} 4 \mathrm{PbBr}_{6} \mathrm{NC}$ dispersion ( $\sim 3 \mathrm{mg} / \mathrm{mL}, \sim 0.001 \mathrm{mmol}$ by $\mathrm{Cs}_{4} \mathrm{PbBr}_{6}$ unit) was added to the vial to initiate the transformation reaction. After the reaction was completed (in $\sim 2$ hours), the solution was centrifuged at $3000 \mathrm{rpm}$ for 3 minutes and the precipitates were discarded. For the transformation reaction resulting $\mathrm{CsPbBr}_{3}$ nanocubes, the $\mathrm{HA}$ was replaced by an equal 
mole amount of OA, and the OctAm was replaced by an equal mole amount of TOAm. The reaction time was prolongated to 3 days in toluene or until the absorption peak of the $\mathrm{Cs}_{4} \mathrm{PbBr}_{6}$ NCs disappeared in the UV-Vis absorption spectrum.

The transformation reaction of $0 \mathrm{D} \mathrm{Cs}_{4} \mathrm{PbI}_{6}$ perovskite $\mathrm{NCs}$ was conducted following a similar procedure as for the case of $\mathrm{Cs}_{4} \mathrm{PbBr}_{6} \mathrm{NCs} \mathrm{PbI}_{2}(15 \mathrm{mg}, 0.033 \mathrm{mmol})$ was added to a mixture solution of hexane $(5 \mathrm{~mL}), \mathrm{HA}(20 \mu \mathrm{L}, 0.16 \mathrm{mmol})$, OAm $(10 \mu \mathrm{L}, 0.03 \mathrm{mmol})$ and OctAm (15 $\mu \mathrm{L}, 0.09 \mathrm{mmol})$ in a scintillation vial and heated to $40^{\circ} \mathrm{C}$. Then, $0.5 \mathrm{~mL} \mathrm{Cs} 4 \mathrm{PbI}_{6} \mathrm{NC}$ dispersion ( $\sim 4 \mathrm{mg} / \mathrm{mL}, \sim 0.001 \mathrm{mmol}$ by $\mathrm{Cs}_{4} \mathrm{PbI}_{6}$ unit) was added to the vial to initiate the transformation reaction. After the reaction was finished (in $\sim 30$ minutes), the solution was centrifuged at 3000 rpm for 3 minutes and the precipitates were discarded. The product $\mathrm{CsPbI}_{3}$ low-dimensional NCs (LD-NCs) were characterized immediately due to its instability.

\section{Synthesis of $\mathrm{PbBr}_{2}$-ligand templates}

For a typical reaction, $\mathrm{PbBr}_{2}(50 \mathrm{mg}, 0.136 \mathrm{mmol})$ was added to a mixture solution of $\mathrm{OA}$ (200 $\mu \mathrm{L}, 0.64 \mathrm{mmol})$ and $\mathrm{OAm}(200 \mu \mathrm{L}, 0.64 \mathrm{mmol})$ in toluene $(5 \mathrm{~mL})$, and reacted under stirring at $60^{\circ} \mathrm{C}$ for 24 hours. OA and OAm were replaced by other ligands with desired molar ratios (1:1:2 molar ratio for the HA:OA:OAm combination) for synthesizing other types of leadligand templates. After the reaction, supernatant was collected and dried for X-ray diffraction measurements.

\section{X-ray diffraction (XRD) measurement}

Powder XRD patterns were obtained on a Bruker D8 Discovery 2D X-ray Diffractometer equipped with a Vantec 500 2D area detector working on $\mathrm{Cu} \mathrm{K \alpha}$ radiation. NC samples dispersed in solution were drop-cast on a glass slide under gentle heating to evaporate the solvent. The X-ray diffraction patterns of the $\mathrm{PbBr}_{2}$-ligand templates were measured on a Bruker D8 Discovery High resolution X-ray Diffractometer equipped with LinxEye 1D detector.

\section{Transmission electron microscopy (TEM) measurement}

TEM images were collected using a JEOL-2100F TEM operating at 200kV. Samples were 
dispersed in hexane and drop-cast on a 300-mesh TEM grid and allowed to dry under ambient conditions.

\section{Absorption, Photoluminescence (PL) and PL excitation (PLE) spectroscopy}

Absorption spectra were measured on an Agilent Cary 8454 UV-Vis Spectrometer. PL and PLE spectra were measured on an Edinburgh Instruments FS5 Spectrofluorometer. NCs were dispersed in hexane for PL measurements. $360 \mathrm{~nm} \mathrm{UV} \mathrm{light} \mathrm{was} \mathrm{used} \mathrm{for} \mathrm{excitation} \mathrm{source} \mathrm{for}$ all PL measurements. All PLE measurements were monitored near the PL peak wavelength. PL QYs were measured using a built-in integrating sphere on the Edinburgh Instruments FS5 Spectrofluorometer.

\section{Time-resolved (TR) PL lifetime measurement}

The TR PL decay curves were measured using Edinburgh Instruments FS5-TCSPC equipped with an EPLED-360 light source. The samples were dispersed in hexane in a quartz cuvette and excited at $360 \mathrm{~nm}$. The lifetime decay was fitted with bi-exponential or tri-exponential decay:

$$
I(t)=\sum_{k} I_{k} \exp \left(-t / \tau_{k}\right)
$$

where $I(t)$ represents the observed PL intensity at the time of $t, I_{k}$ and $\tau_{k}$ represent the amplitude and lifetime of exponential decay component of k, respectively. Average lifetimes were calculated by the following equation:

$$
\tau_{\text {avg }}=\frac{\sum_{k} I_{k} \tau_{k}^{2}}{\sum_{k} I_{k} \tau_{k}}
$$

Relative percentage of the decay component of $i\left(P_{i}\right)$ was calculated by the following equation:

$$
P_{i}=\frac{I_{i} \tau_{i}}{\sum_{k} I_{k} \tau_{k}}
$$




\section{Supporting figures and tables}
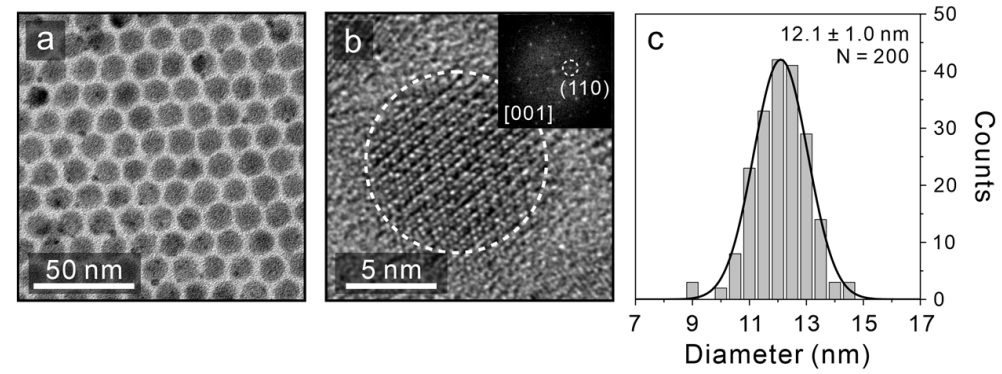

Figure S1. (a) TEM image of 0D Cs4 $\mathrm{PbBr} 6$ perovskite NCs. (b) High-resolution (HR) TEM image of the $\mathrm{Cs}_{4} \mathrm{PbBr}_{6}$ NCs. Inset: the fast Fourier transform (FFT) of the image. The FFT reveals that the HR-TEM image is viewed from the [001] direction of the hexagonal $\mathrm{Cs}_{4} \mathrm{PbBr}_{6}$ crystal structure. (c) The histogram of the diameter of the $\mathrm{Cs}_{4} \mathrm{PbBr}_{6} \mathrm{NCs}_{\text {(grey }}$ bars) and the corresponding normal distribution function (black line). 
Table S1. TR-PL fitting parameters for $\mathrm{CsPbBr}_{3} \mathrm{NWs}$ and nanocubes, and $\mathrm{CsPbI}_{3} \mathrm{LD}-\mathrm{NCs}$.

\begin{tabular}{cccc}
\hline Sample & $\boldsymbol{\tau}$ (ns) & Rel. percentage (\%) & $\overline{\boldsymbol{\tau}}$ (ns) \\
\hline \multirow{3}{*}{$\mathrm{CsPbBr}_{3} \mathrm{NWs}$} & 1.5 & 15.6 & \\
& 4.8 & 57.0 & 7.1 \\
& 15.0 & 27.4 & \\
\hline \multirow{2}{*}{$\mathrm{CsPBr} 3$ Nanocubes } & 3.7 & 8.0 & \\
& 19.0 & 41.4 & 57.0 \\
& 96.6 & 50.6 & \\
\hline \multirow{2}{*}{$\mathrm{CsPI}_{3}$ LD-NCs } & 10.8 & 33.9 & \multirow{2}{*}{30.8} \\
\hline
\end{tabular}



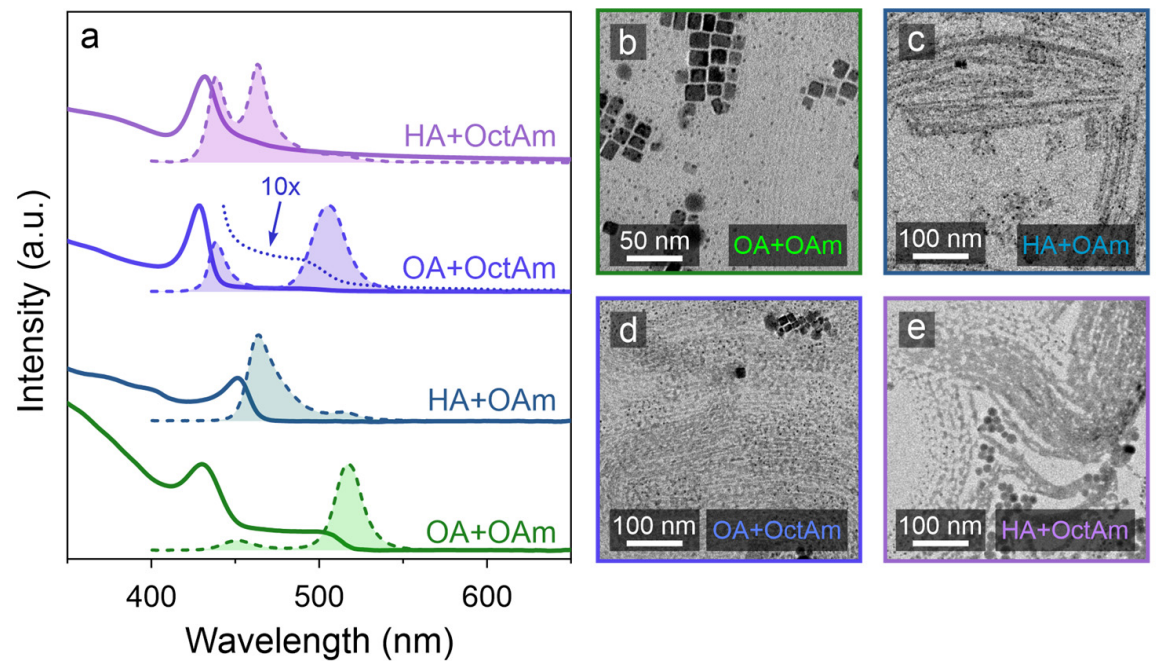

Figure S2. (a) Absorption (solid lines) and PL (dashed lines) spectra of the transformation reactions using different ligand combinations. The dotted blue line represents the enlarged absorption spectrum. (b-e) TEM images of the transformation products using $\mathrm{OA}+\mathrm{OAm}$, $\mathrm{HA}+\mathrm{OAm}, \mathrm{OA}+\mathrm{OctAm}$, and $\mathrm{HA}+\mathrm{OctAm}$, respectively. 
Table S2. XRD fitting results of the $\mathrm{PbBr}_{2}$-ligand intermediates showed in Figure 3.

\begin{tabular}{|c|c|c|c|c|}
\hline Ligand & $\begin{array}{c}\text { Assigned peak } \\
(00 n)\end{array}$ & $\begin{array}{c}2 \theta \\
\text { (degree) }\end{array}$ & $\begin{array}{c}d \text {-spacing } \\
(\AA)\end{array}$ & $\begin{array}{c}\text { Average interlayer } \\
d \text {-spacing }(\AA)\end{array}$ \\
\hline \multirow{5}{*}{$\mathrm{OA}+\mathrm{OAm}$} & $(002)$ & 4.24 & 20.8 & \multirow{5}{*}{$41.7 \pm 0.04$} \\
\hline & $(003)$ & 6.36 & 13.9 & \\
\hline & $(004)$ & 8.47 & 10.4 & \\
\hline & $(005)$ & 10.59 & 8.349 & \\
\hline & $(006)$ & 12.71 & 6.963 & \\
\hline \multirow{4}{*}{$\mathrm{HA}+\mathrm{OAm}$} & $(002)$ & 4.80 & 18.4 & \multirow{4}{*}{$37.0 \pm 0.1$} \\
\hline & $(003)$ & 7.17 & 12.3 & \\
\hline & $(004)$ & 9.57 & 9.24 & \\
\hline & $(005)$ & 11.93 & 7.416 & \\
\hline \multirow{8}{*}{$\mathrm{OA}+\mathrm{OctAm}$} & $(001)$ & 3.06 & 28.9 & \multirow{4}{*}{$29.0 \pm 0.1$} \\
\hline & $(002)$ & 6.08 & 14.5 & \\
\hline & $(003)$ & 9.11 & 9.70 & \\
\hline & $(004)$ & 12.17 & 7.269 & \\
\hline & $(001)$ & 4.12 & 21.4 & \multirow{4}{*}{$21.5 \pm 0.01$} \\
\hline & $(002)$ & 8.23 & 10.7 & \\
\hline & $(003)$ & 12.37 & 7.15 & \\
\hline & $(004)$ & 16.52 & 5.36 & \\
\hline \multirow{4}{*}{$\mathrm{HA}+\mathrm{OctAm}$} & $(001)$ & 3.94 & 22.4 & \multirow{4}{*}{$22.5 \pm 0.03$} \\
\hline & $(002)$ & 7.87 & 11.2 & \\
\hline & $(003)$ & 11.81 & 7.491 & \\
\hline & $(004)$ & 15.76 & 5.620 & \\
\hline \multirow{4}{*}{$\mathrm{HA}+\mathrm{OA}+\mathrm{OctAm}$} & $(001)$ & 3.64 & 24.3 & \multirow{4}{*}{$24.3 \pm 0.04$} \\
\hline & $(002)$ & 7.25 & 12.2 & \\
\hline & $(003)$ & 10.91 & 8.106 & \\
\hline & $(004)$ & 14.58 & 6.070 & \\
\hline
\end{tabular}



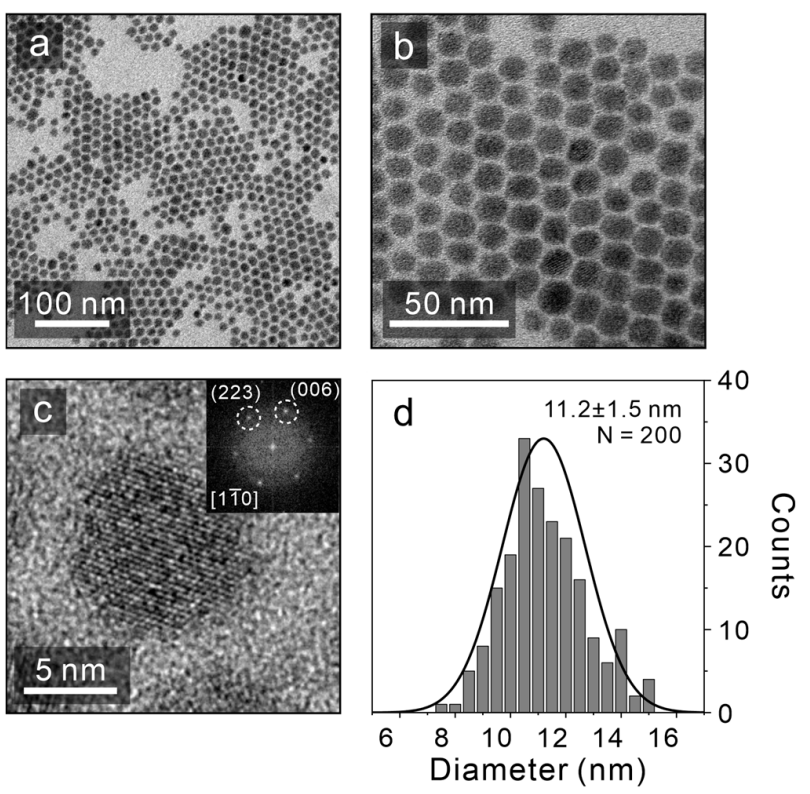

Figure S3. (a, b) TEM images of the 0D Cs4 $\mathrm{PbI}_{6}$ perovskite NCs. (c) HR-TEM image of the $\mathrm{Cs}_{4} \mathrm{PbI}_{6} \mathrm{NCs}$. Inset: the corresponding fast Fourier transform, suggesting that the image is viewed from the [1 $1 \overline{1} 0]$ direction of the hexagonal $\mathrm{Cs}_{4} \mathrm{PbI}_{6}$ crystal structure. (d) The histogram of the diameter of the $\mathrm{Cs}_{4} \mathrm{PbI}_{6} \mathrm{NCs}$ (grey bars) and the corresponding normal distribution function (solid line). 


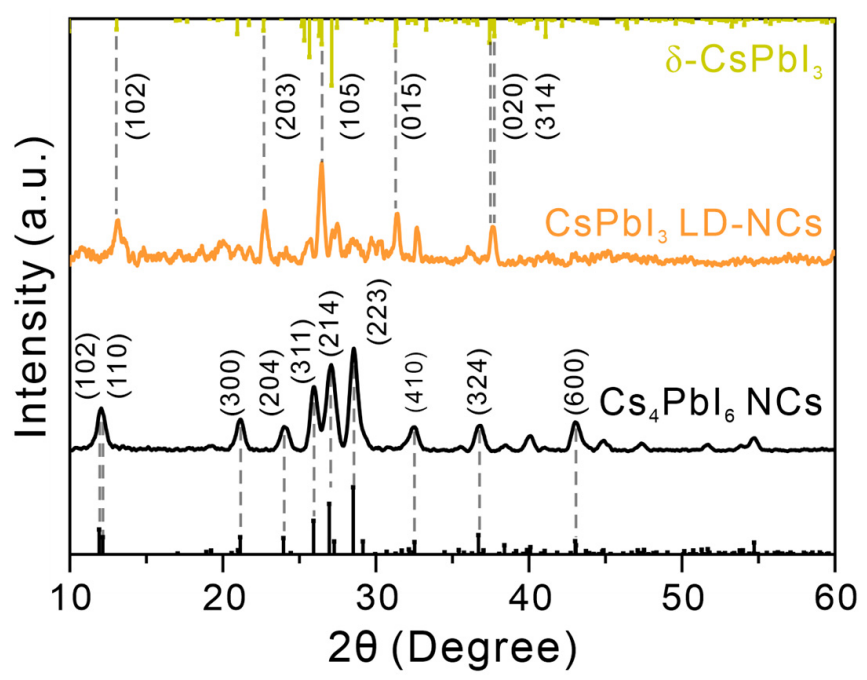

Figure S4. XRD patterns of the starting $0 \mathrm{D} \mathrm{Cs}_{4} \mathrm{PbI}_{6}$ perovskite $\mathrm{NCs}$ (bottom) and the decomposed $\mathrm{CsPbI}_{3} \mathrm{LD}_{-\mathrm{NCs}}$ upon drying (top). Bars represent the standard diffraction patterns. Upon drying, the $\mathrm{CsPbI}_{3}$ LD-NCs quickly decomposed to an orthorhombic $\delta$ phase. 


\section{Reference:}

1. Akkerman, Q. A.; Park, S.; Radicchi, E.; Nunzi, F.; Mosconi, E.; De Angelis, F.; Brescia, R.; Rastogi, P.; Prato, M.; Manna, L., Nearly Monodisperse Insulator $\mathrm{Cs}_{4} \mathrm{PbX} \mathrm{X}_{6}$ (X $=\mathrm{Cl}$, Br, I) Nanocrystals, Their Mixed Halide Compositions, and Their Transformation into $\mathrm{CsPbX}_{3}$ Nanocrystals. Nano Letters 2017, 17 (3), 1924-1930. 